\title{
CARACTERIZACIÓN MORFOLÓGICA Y GENÉTICA DE ETNOVARIEDADES DE LA YUCA Manihot esculenta, Cratnz, EN SEIS LOCALIDADES DE LA CUENCA BAJA DEL Río UCAYALI-PERÚ
}

\author{
Jose MEJÍA ${ }^{1}$, Rosa ELIAS-DA-SILVA², Kember MEJÍA ${ }^{3}$, Carmen GARCÍA-DÁVILA \\ 1 Laboratorio de Biología y Genética Molecular - LBGM. Instituto de Investigaciones de la Amazonía Peruana, IIAP, \\ Carretera Iquitos-Nauta km. 4.5, Iquitos, Perú, e-mail: edu 345@hotmail.com \\ 2 Universidad Federal de Santa Catarina, Programa de post-graduación en biología de hongos, algas y plantas, \\ Florianópolis, Santa Catarina, Brasil. \\ 3 Programa de Investigación en Biodiversidad Amazónica-PIBA, Instituto de Investigaciones de la Amazonía Peruana-IIAP. \\ Av. A. Quiñonez, Km 2.5 Iquitos-Perú.
}

\section{RESUMEN}

La caracterización morfológica y genética de la yuca Manihot esculenta fue realizada mediante la evaluación de 22 descriptores morfológicos y 12 marcadores moleculares (microsatélites). La evaluación de los caracteres morfológicos, mostraron que si bien existe una sobre posición de caracteres entre algunas etnovariedades, siempre es posible diferenciarlos por al menos un carácter, validando morfológicamente las 26 etnovariedades evaluadas. El descriptor con mayor plasticidad fue la altura de la primera ramificación (APR) con coeficiente de variación (CV) de 89.3; mientras que el descriptor más estable para las 26 etnovariedades fue la longitud del lóbulo central de la hoja (LLP) con CV de 0.9. El análisis genético muestra que las etnovariedades están agrupadas en cinco grupos bien definidos, mientras 92 individuos (71.9\%) compartieron su genotipo con uno o más individuos, en muchos de los casos de otras etnovariedades. El análisis de las cuatro variedades más comercializadas (señorita, cogollo morado, amarilla, piririca), mostraron que algunos individuos comparten genotipos con otros individuos de su misma etnovariedad en las seis localidades analizadas. La excepción fue la etnovariedad "señorita" proveniente de la comunidad de San José de Paranapúra, que compartieron el mismo genotipo que otros individuos de la etnovariedad "cogollo morado" de Chingana. Esto podría significar que: i) los pobladores de estas localidades están confundiendo las etnovariedades, ii) que la similitud fenotípica observada en la yuca no es necesariamente producto de la similitud genotípica, o iii) que Manihot esculenta, a pesar de ser una especie con muchos años de domesticación encierra todavía una gran variabilidad genética.

PALABRAS CLAVE: Descriptores morfológicos, marcadores moleculares, variabilidad genética, genotipos, Manihot esculenta, yuca.

\section{MORPHOLOGICAL AND GENETIC CHARACTERIZATION OF CASSAVA LANDRACES Manihot esculenta, Cratnz IN SIX LOCATIONS IN THE LOWER RIVER BASIN UCAYALI - PERU}

\begin{abstract}
The morphological and genetic characterization of 26 cassava landraces from six locations in the lower basin of the Ucayali River was analysed using 22 morphological descriptors (15 qualitative and 7 quantitative) and twelve molecular markers (microsatellites). Morphological results showed that while there is an overlapping of characters between some landraces, it is always possible to differentiate them by at least one character, validating morphologically the 26 landraces evaluated. The descriptor with greater plasticity was the height of the first branch with coefficient of variation (CV) of 89.3. While the most stable descriotor among the 26 landraces was the length of the central lobe with $0.9 \mathrm{CV}$. The genetic analysis showed that landraces are grouped into five major groups, while a great number of individuals shared their genotype with one or more individuals (92 individuals $=71.9 \%$ ), in many cases with individuals of other landraces. The analysis of the four most traded varieties showed that some individuals share genotypes with other individuals of the same landrace in six geographic locations analyzed. The exception was the etnovariedad "señorita" from the community of San José de Paranapura, which shared the same genotype as other individuals of the etnovariedad "cogollo morado" of Chingana. This may mean that: i) the local people are confusing landraces in the study localities, ii) the observed phenotypic similarity in cassava is not necessarily a product of genotypic similarity, or iii) Manihot esculenta, despite being a species with many years of domestication still holds a great genetic variability in crops.
\end{abstract}

KEYWORDS: Morphological descriptors, molecular markers, genetic variability, genotypes, Manihot esculenta, manioc. 


\section{INTRODUCCIÓN}

En la Amazonía peruana una situación común de la agricultura tradicional, es la existencia de diferentes especies y variedades plantas cultivadas, en una misma chacra (Boster, 1983; Elias \& Mckey, 2000). El cultivo de subsistencia más importante en estas chacras es la yuca Manihot esculenta de la que se distinguen un gran número de variedades locales (etnovariedades), que son identificadas por los agricultores con un nombre común o singular, el cual casi siempre está basado en características morfológicas, lugar de origen de las variedades o nombres de personas que introdujeron la variedad a la comunidad (Elias \& Mckey, 2000; Mkumbira et al., 2003). La diversidad morfológica y genética de esta especie ha sido caracterizada en términos de variedades cultivadas, estimándose alrededor de 7000 variedades encontradas en todo el mundo (Mühlen et al., 2000), en su mayoría etnovariedades, mantenidas por agricultores tradicionales (Hershey, 1994; Emperaire \& Pinton, 1998).

En la región de estudio, los sistemas agrícolas tradicionales tienen como especie fundamental a la yuca Manihot esculenta, por presentar una elevada diversidad intra e intervarietal, sea dentro de los campos de cultivo, como dentro o entre comunidades (Peroni \& Martins, 2000). Es por ello que la yuca viene siendo objeto de numerosos estudios de caracterización morfológica (descriptores) y molecular (marcadores moleculares), que buscan conocer la gran diversidad que encierra esta especie. El conocimiento de la diversidad genética de los cultivos, en cualquier región del mundo, permite aportar información básica en la toma de decisiones para propósitos de mejoramiento y conservación de la especie (Lobo, 2004). En este sentido, el presente estudio tiene como objetivo la caracterización morfológica y genética de las etnovariedades de yuca en seis localidades de la cuenca baja del rio Ucayali, mediante el empleo de descriptores morfológicos y uso de marcadores microsatélites.

\section{MATERIALES Y MÉTODOS}

\section{Área de colecta de material biológico}

La colecta del material biológico se realizó en seis comunidades, pertenecientes al Distrito de Sapuena, Provincia de Requena, región Loreto, Amazonía peruana (Figura 1). Dos de las comunidades estaban ubicadas en zonas de tierra firme: Bagazán (UTM 9477981, 664235) y Flor de Castaña (UTM 9472663, 655402); en tanto que las cuatro restantes estaban ubicadas en zonas inundables: San José de Paranapúra (UTM 9500610 , 671041), Chingana (UTM 9478201, 66195), Sapuena (UTM 9472713, 651941) y Jorge Chávez (UTM 9471862, 649812).

\section{Análisis morfológico}

Fueron analizados un total de cinco individuos por variedad en cada una las localidades evaluadas. Los caracteres morfológicos evaluados estaban conformados por 15 caracteres cualitativos y siete cuantitativos, todos ellos publicados y validados por Fukuda \& Guevara en el 1998.

\section{Análisis molecular SSR}

Extracción - amplificación del ADNy determinación del peso de los alelos

La extracción de ADN fue realizada a partir de tejido foliar mediante el método CTAB (Doyle \& Doyle, 1987). La variabilidad genética fue determinada a partir de un total de 12 locus microsatélites, ocho de ellos amplificados utilizando los primers desarrollados por Chavarriaga-Aguirre et al. (1998): GA-5, GA-12, GA-21, GA-126, GA131, GA-134, GA-136, GA-140 y cuatro desarrollados por Mba et al. (2001): SSRY4, SSRY9, SSRY20, SSRY21. La reacción de amplificación estuvo compuesta de $2.0 \mu 1$ de Buffer (1x); $0.5 \mu \mathrm{l}$ de $\mathrm{MgCl}_{2}(1.25 \mathrm{mM}) ; 0.2 \mu \mathrm{l}$ de cada dNTP $(1 \mathrm{mM}) ; 0.4 \mu 1$ de cada primer; $0.08 \mu 1$ de taq polimerasa $(5 \mathrm{U} / \mu \mathrm{l}), 5.82 \mu \mathrm{l}$ de agua mili-Q y $1 \mu \mathrm{lde}$ ADN $(100 \mathrm{ng} / \mu \mathrm{l})$. Las condiciones de temperatura fueron: una denaturación inicial a $96^{\circ} \mathrm{C}$ por $2 \mathrm{~min}$., seguido de 30 ciclos conformados por Denaturación a $94^{\circ} \mathrm{C}$ por $1 \mathrm{~min}$.; Hibridación a $56.6^{\circ} \mathrm{C}$ por $1 \mathrm{~min}$.; y Extensión a $72^{\circ} \mathrm{C}$ por $1 \mathrm{~min}$. Seguida de una extensión final a $72^{\circ} \mathrm{C}$ por $30 \mathrm{seg}$. El peso de los alelos fue determinado usando el software Peak Scanner versión 1.0 (https://products.appliedbio systems.com).

\section{Tratamiento de datos}

\section{Datos morfológicos}

La matriz de datos morfológicos se organizó en caracteres cuantitativos y cualitativos (en escala nominal) y fueron analizados mediante estadística descriptiva. El análisis de los datos morfológicos se realizó en el programa Statistica versión 8.0. Por cada carácter cuantitativo en cada etnovariedad fue estimado el promedio, la desviación estándar, el coeficiente de variación, valor mínimo, valor máximo. En tanto que para los caracteres cualitativos se determinó la moda y la frecuencia. 
FOLIA

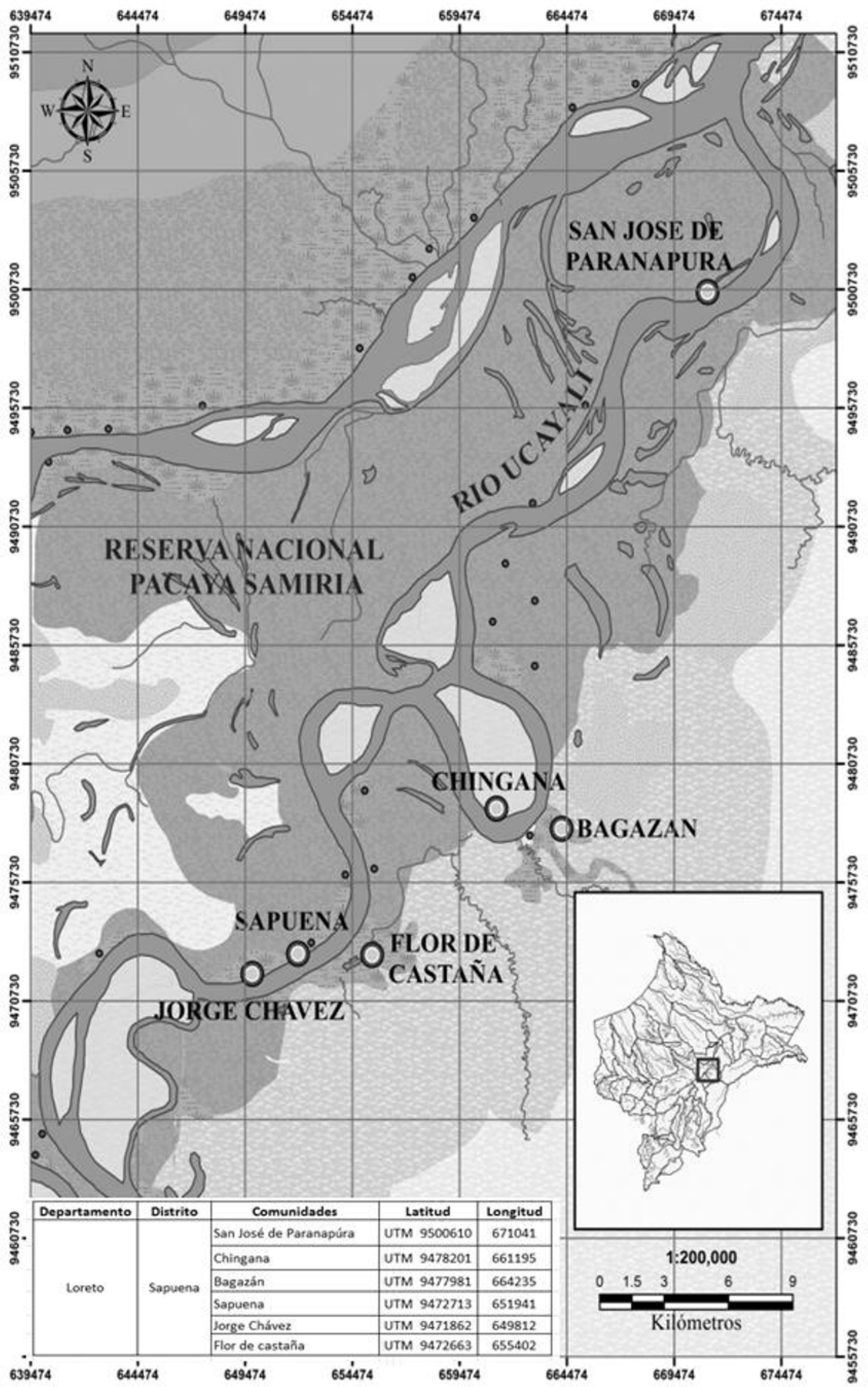

Figura 1. Mapa de ubicación de los lugares de colecta de los individuos de yuca Manihot esculenta Cratnz. 
Tabla 1. Etnovariedades de yuca colectadas en seis comunidades de la cuenca baja del rio Ucayali.

\begin{tabular}{|c|c|c|c|c|c|c|c|}
\hline \multirow{2}{*}{ Etnovariedad } & \multirow{2}{*}{ Código } & \multicolumn{6}{|c|}{ Localidades } \\
\hline & & $\begin{array}{l}\text { SAN JOSE DE } \\
\text { PARANAPÚRA }\end{array}$ & CHINGANA & BAGAZÁN & SAPUENA & $\begin{array}{l}\text { FLOR DE } \\
\text { CASTAÑA }\end{array}$ & $\begin{array}{l}\text { JORGE } \\
\text { CHÁVEZ }\end{array}$ \\
\hline señorita & SR & $x$ & $x$ & & $x$ & $x$ & $x$ \\
\hline pinsha rumo & PS & & & & & & $x$ \\
\hline piririca & $\mathrm{PI}$ & $x$ & $x$ & & $x$ & $x$ & $x$ \\
\hline palo negro & PN & & & & & $x$ & $x$ \\
\hline paloma rumo & PL & $\mathrm{x}$ & & & & & $x$ \\
\hline tijerilla & TJ & & & & $x$ & & \\
\hline yuca blanca & $\mathrm{BL}$ & & & & $x$ & & \\
\hline lacre rumo & LC & & & & $x$ & & \\
\hline cogollo morado & $\mathrm{CO}$ & & $x$ & & $x$ & $x$ & \\
\hline bolsa rumo & BO & & & & $x$ & & \\
\hline arahuana rumo & AR & & & & $x$ & $x$ & \\
\hline wasahí rumo & WA & & & & $x$ & & \\
\hline cola de zorro & ZO & $x$ & & & & & \\
\hline sarayacu & SA & $x$ & & & & & \\
\hline yuca vidrillo & VI & $x$ & & & & & \\
\hline concha rumo & CR & & $x$ & & & & \\
\hline yuca tigre & TG & & $x$ & & & & \\
\hline señorita gigante & SG & & $x$ & & & & \\
\hline arpón rumo & AP & & & $x$ & & & \\
\hline tres mesina & TR & & & $x$ & & & \\
\hline piririca del alto & PA & & & $x$ & & & \\
\hline piririca del bajo & PB & & & $x$ & & & \\
\hline yuca pucallpina & PU & & & $x$ & & & \\
\hline tijerilla morada & TM & & & & & $x$ & \\
\hline ungurahui & UN & & & & $x$ & $x$ & \\
\hline yuca amarilla & AM & $x$ & $x$ & $x$ & & $x$ & \\
\hline
\end{tabular}




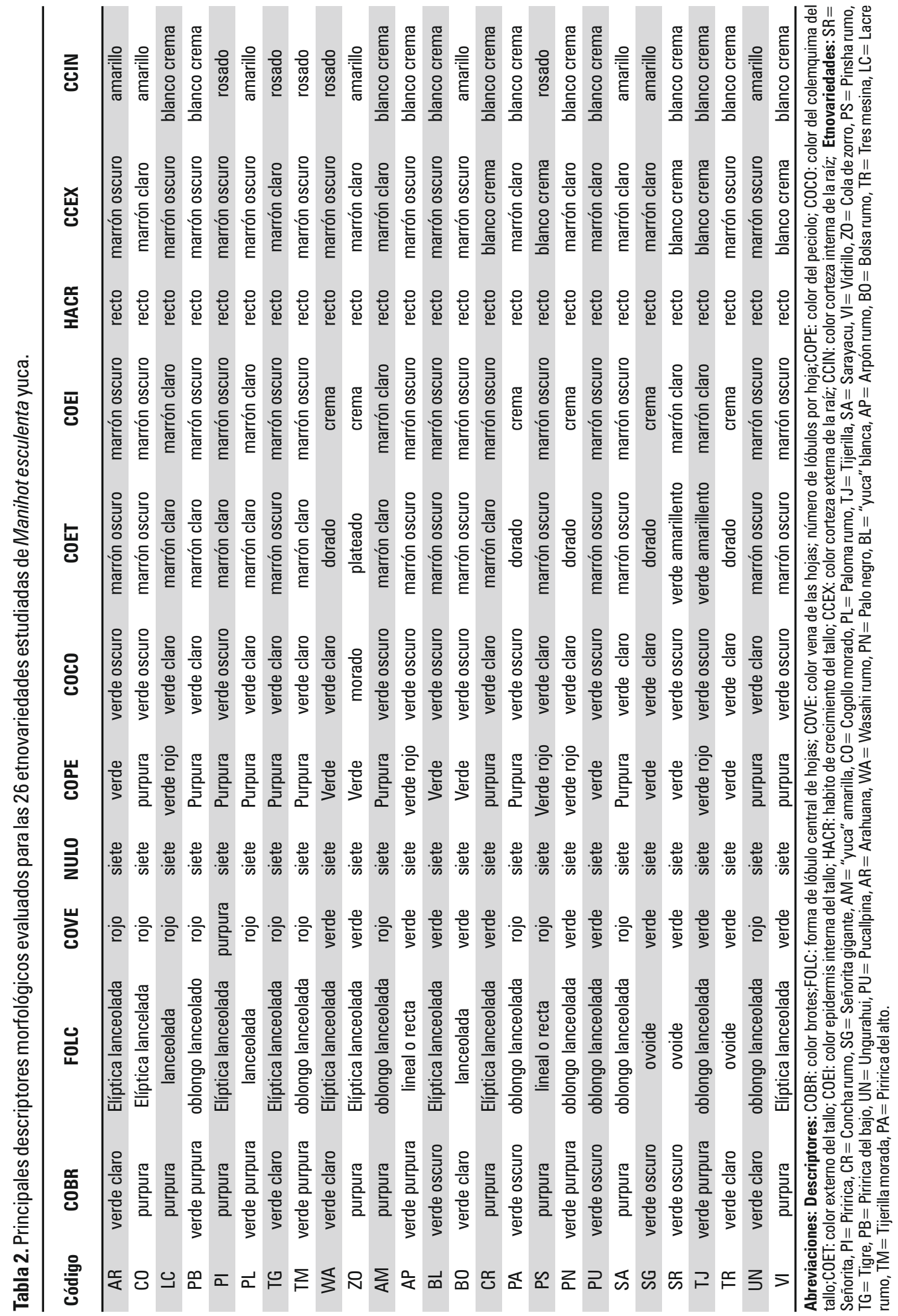




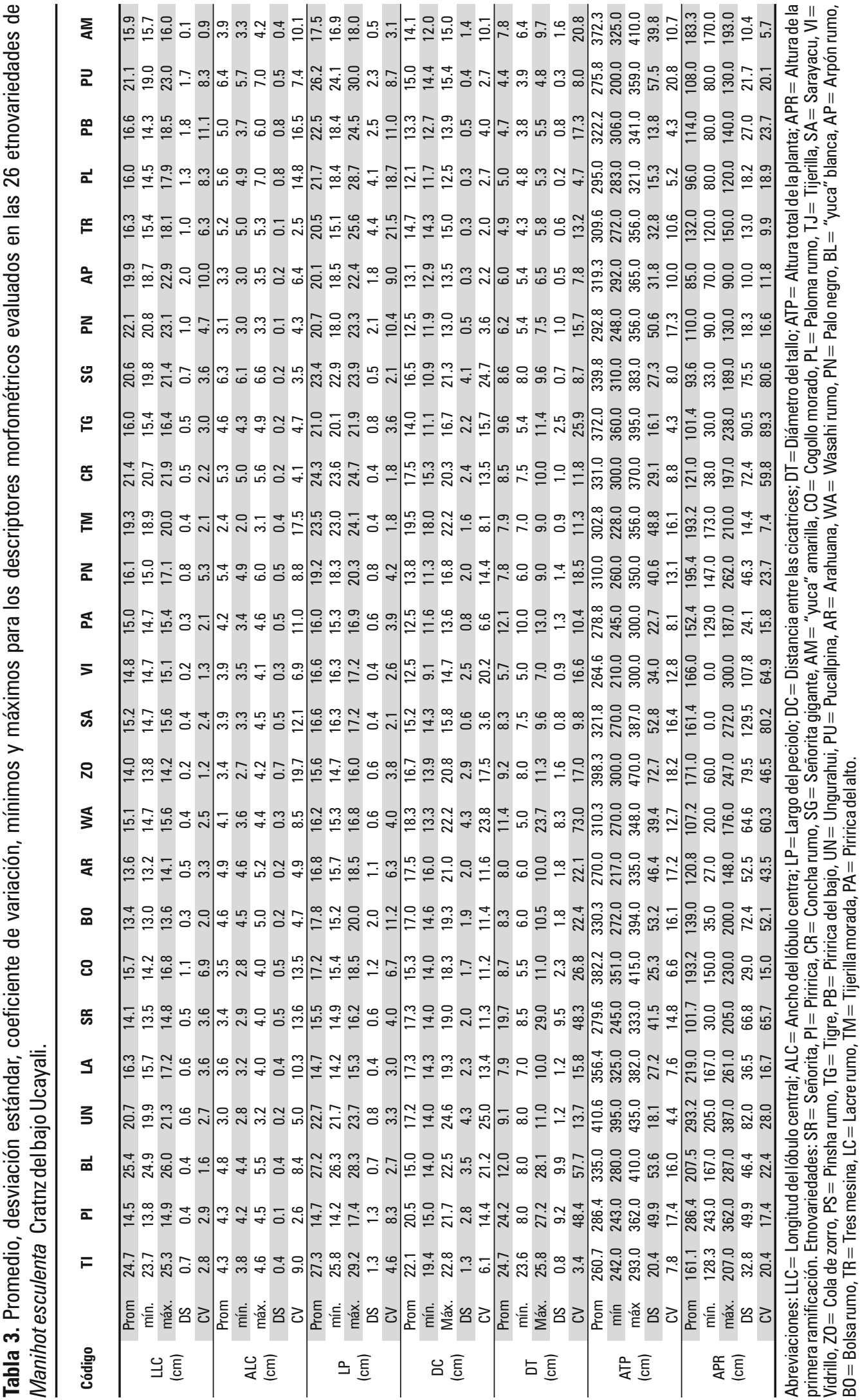




\section{Datos moleculares}

La estimación del número y la frecuencia de alelos por locus, así como el polimorfismo de los alelos, fueron calculadas con la ayuda del software GenAlex versión 6.0 (Peakall \& Smouse, 2005). La distancia genética de Nei (1972) entre las etnovariedades comerciales (aquellas que presentaron la misma denominación en por lo menos tres comunidades; "piririca", "yuca amarilla", "cogollo morado" y "señorita"); fue estimada con la ayuda del software Genetix versión 4.05 (Belkhir et al., 2004). Las relaciones genéticas entre las 26 etnovariedades, así como entre las cuatro comerciales (señorita, cogollo morado, piririca y amarilla) fueron determinadas utilizando el software PHYLIP versión 3.5 (Felsenstein, 1993). Las etnovariedades de cada comunidad fueron tratadas como unidades taxonómicas operacionales (OTUs) y el soporte estadístico para los nudos correspondió a los valores de bootstrap obtenidos con 1000 réplicas.

\section{RESULTADOS Y DISCUSIÓN}

\section{Análisis morfológico}

Se logró caracterizar morfológicamente un total de 26 etnovariedades de yuca para las seis localidades estudiadas (Tabla 1), ocho de estas etnovariedades estuvieron presentes en más de una comunidad ("señorita", "piririca", "yuca amarilla", "cogollo morado", "palo negro", "paloma rumo", "arahuana rumo" y "ungurahui rumo"). En la zona de estudio se han reportado 38 variedades locales de yuca; los resultados confirman la continua disminución y pérdida de etnovariedades reportadas para esta zona, desde el año 1984 a la actualidad (Inga \& Parodi 2001; Gonzales, 2002). Esta pérdida de etnovariedades podría estar relacionada a la inviabilidad de estacas de las diferentes etnovariedades como consecuencia de las crecientes excepcionales del río Ucayali en este periodo de tiempo, especialmente el año 2012. Así como, por el desplazamiento o perdida de algunas etnovariedades por selección y masificación del cultivo de otras etnovariedades con características específicas deseadas (color de pulpa, calidad de productos derivados, etc.) para el comercio en los distintos mercados de consumo.

\section{Variables Cualitativas}

Los caracteres cualitativos evaluados fueron muy variables entre las etnovariedades, siendo que los más variables fueron: la coloración de los brotes, el color de los peciolos y el color de la Corteza interna y externa de las raíces. Sin embargo caracteres como: el número de lóbulos por hoja y el hábito de crecimiento de los tallos no presentaron ninguna variación entre las etnovariedades evaluadas (Tabla 2). Los resultados demuestran que a pesar de haberse encontrado una sobreposición parcial de caracteres (colores y formas) entre algunas etnovariedades, todas ellas se diferenciaron en al menos un carácter, demostrando que las 26 etnovariedades evaluadas son válidas. Numerosos estudios sugieren que algunos descriptores como el color de la hoja apical, la forma del lóbulo central, número de lóbulos por hoja, color del pecíolo, color del tallo, color de la corteza del tallo, color de la corteza de la raíz y color de la pulpa de la raíz presentan baja influencia ambiental, por lo que son características sinópticas importantes para la identificación y diferenciación de variedades de yuca Manihot esculenta (Peroni et al., 1999; Lobo, 2004; Acosta et al., 2006).

\section{Variables cuantitativas}

La caracterización morfométrica (Tabla 3), mostró que los descriptores con mayor plasticidad (variación) fueron la altura de la primera ramificación (APR) y la distancia entre las cicatrices de las hojas (DC) con Coeficientes de variación (CV) de 89.3 y 73.00 respectivamente; mientras que los descriptores más estables (menos variables) fueron la longitud del lóbulo central (LLP) y el largo del peciolo (LP) con CV de 0.9 y 1.8 respectivamente.

\section{Análisis molecular}

Las etnovariedades de Manihot esculenta evaluadas en este estudio presentaron una alta variabilidad genética en todos los microsatélites evaluados. Fueron encontrados un total de 47 alelos en las 12 loci microsatélites evaluadas (tabla 4). La mayor diversidad alélica fue encontrada en el locus Ga-126 (9 alelos), y la más baja en los loci Ga-5, Ga-134 y Ga-12 (2 alelos cada uno), con un promedio de 3.75 alelos por locus. Los pesos de los alelos variaron entre los 93 pb y 319 pb. La heterosigocidad observada fue mayor que la heterosigocidad esperada (media $\mathrm{Ho}=0.599$ y media $\mathrm{He}=0.407$ ). Este elevado polimorfismo puede deberse a que Manihot esculenta a pesar de tener una reproducción asexual por estacas, también podría estar combinada con ciclos de reproducción sexual, permitiendo la recombinación de la variación actual y como tal la generación de nuevas formas o combinaciones. 
Tabla 4. Número de alelos y heterocigocidad observada y esperada para cada locus de Manihot esculenta Cratnz.

\begin{tabular}{cccc}
\hline & $\begin{array}{c}\text { Alelos por } \\
\text { locus }\end{array}$ & $\mathbf{H}_{\mathbf{o}}$ & $\mathbf{H}_{\mathbf{e}}$ \\
\hline GA-5 & 2 & 0.616 & 0.383 \\
\hline GA-136 & 3 & 0.728 & 0.451 \\
\hline GA-126 & 9 & 0.877 & 0.613 \\
\hline GA-21 & 3 & 0.506 & 0.354 \\
\hline SSRY20 & 4 & 0.694 & 0.500 \\
\hline GA-134 & 2 & 0.475 & 0.313 \\
\hline GA-131 & 5 & 0.780 & 0.521 \\
\hline GA-140 & 4 & 0.418 & 0.297 \\
\hline SSRY4 & 6 & 0.680 & 0.522 \\
\hline GA-12 & 2 & 0.025 & 0.020 \\
\hline SSRY21 & 3 & 0.555 & 0.361 \\
\hline SSRY9 & 4 & 0.829 & 0.550 \\
\hline Promedio & 47 & 0.599 & 0.407 \\
\hline Desviación & - & 0.230 & 0.158 \\
estándar & & & \\
\hline
\end{tabular}

Es decir, la variabilidad a nivel de chacras no es estática y con el paso del tiempo hay genotipos que desaparecen y otros que se incorporan al sistema de producción del agricultor (Iglesias \& Hernández, 1994; Alzate et al., 2009). Esto podría ser una posible explicación al gran número de etnovariedades encontradas en un área geográfica restricta como la estudiada.

\section{Relación genética entre las etnovariedades}

Los valores de distancia genética (Nei, 1972) mostraron que las etnovariedades más cercanas fueron "yuca amarilla" y "tigre" (0.01), seguida de "tres mesina" y "señorita gigante" (0.03); mientras que las más distantes fueron "sarayacu y tijerilla" (0.99).

Esto fue confirmado por los resultados de relacionamiento genético (análisis de Neighbor Joining) que demuestra que las 26 etnovariedades están conformando cinco grupos genéticos bien definidos (Figura 2). El grupo 1 está formado por tres subgrupos (subgrupo 1=TM, TJ, LC, AP, WA; subgrupo $2=\mathrm{BO}, \mathrm{PA}, \mathrm{CO}$ y subgrupo $3=\mathrm{ZO}, \mathrm{CR}$ ); mientras que el grupo cuatro está formado por dos subgrupos (subgrupo $1=\mathrm{PU}, \mathrm{BL}$, PN y subgrupo $2=$ UN, SA, TG, AM); en tanto que los grupos 3, 2 y 5 no estaban subdivididos. Cuando analizamos estas etnovariedades tanto desde el punto de vista de los caracteres morfológicos, como de los genéticos, encontramos que no existe un $100 \%$ de correspondencia entre estos dos grupos de variables, demostrándose que etnovariedades genéticamente cercanas, no siempre son las más semejantes desde el punto de vista morfológico. Esto podría deberse a que la caracterización mediante descriptores morfológicos (cuantitativos y cualitativos) no siempre refleja la variación real, debido a que el fenotipo está determinado parcialmente por la información genética del individuo, e influenciado por el ambiente donde se desarrolla (Valadez et al., 2001; Vaz et al., 2004).

El análisis de las cuatro etnovariedades comerciales en las seis localidades de la cuenca baja del Ucayali mostró que individuos reconocidos por los agricultores como etnovariedades diferentes, presentaron el mismo genotipo (2 individuos de "señorita" provenientes de la localidad de San José de Paranapura con cuatro individuos de "cogollo morado" de la localidad de Chingana). Esto puede tener dos interpretaciones: i) que la similitud fenotípica no es necesariamente producto de la similitud genotípica, siendo así que diferentes pool de genes pueden generar fenotipos similares (Demey et al., 2003), como fue registrado entre variedades de maíz Zea mays (Ben-Har et al., 1995) y Musa sp. (Ortiz, 1997; Crouch, 2000). O en su defecto, ii) que el agricultor haya confundido las etnovariedades en el momento de sembrar, ya que no siempre es posible la diferenciación de especies basada solamente en características morfológicas (Nye, 1991), más aun entre variedades dentro de una misma especie, donde existe un gran porcentaje de caracteres morfológicos sobrepuestos y plasticidad morfológica.

La presencia de dos genotipos compartidos entre un buen número de individuos dentro de las etnovariedades comerciales "piririca" y "señorita" provenientes de diferentes comunidades, muestra que las etnovariedades dentro de cada chacra y cada localidad, están sujetas a la acción de varias fuerzas evolutivas, como la selección artificial realizada por los agricultores locales, el cruzamiento entre diferentes etnovariedades y la migración de genotipos de un lugar a otro, que se produce debido al constante intercambio de material de siembra (estacas) entre los pobladores, sin una aparente relación con la distancia geográfica de las comunidades, esto ya fue observado en otros países en este tipo de cultivo (Fregene et al., 2001; Pujol, 2005; Sardos et al.; 2008). 


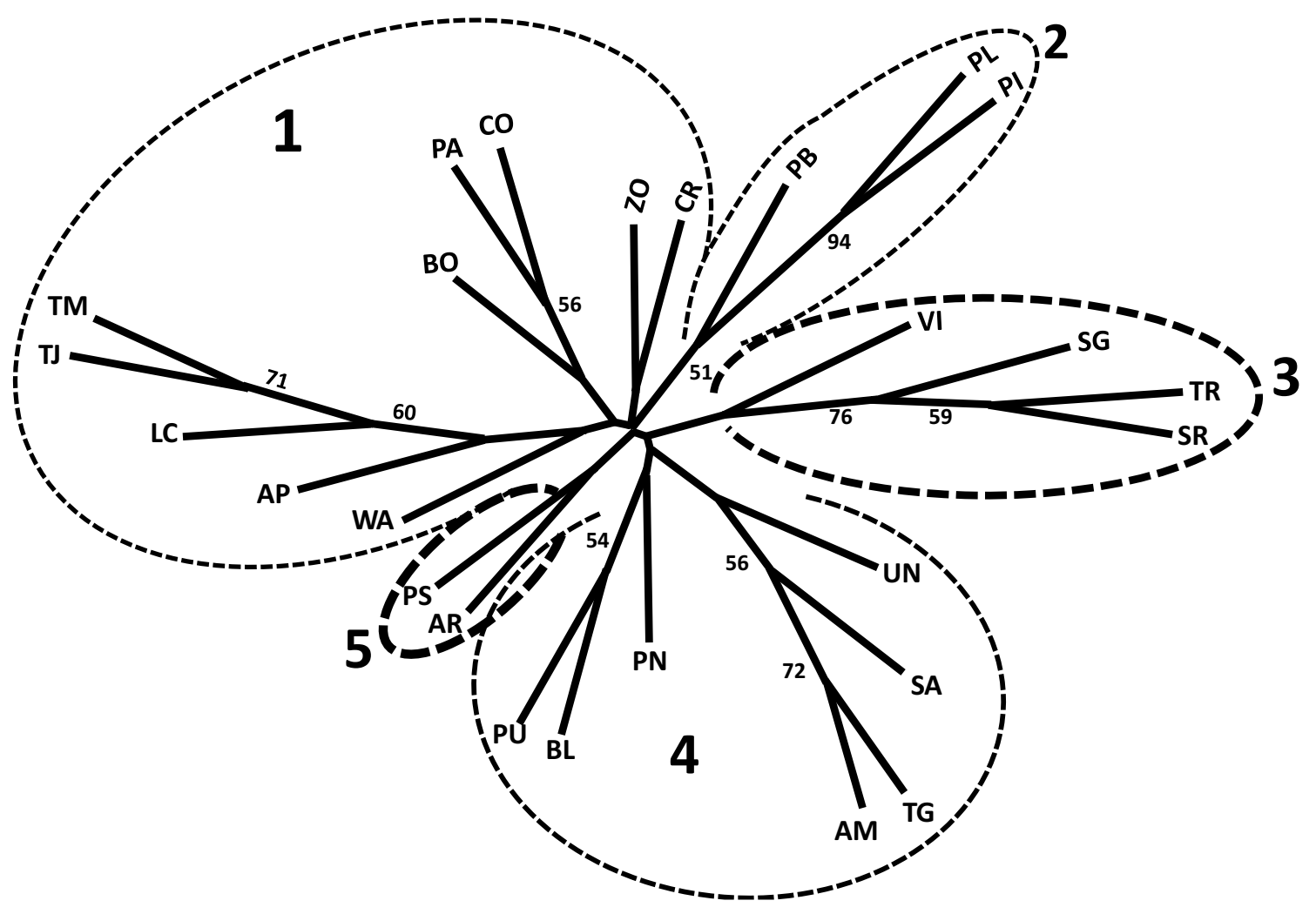

Figura 2. Etnovariedades de Manihot esculenta, Cratnz proveniente de seis comunidades de la cuenca baja del rio Ucayali. Abreviaciones: $\mathrm{SR}=$ "señorita", $\mathrm{PI}=$ "Piririca", $\mathrm{CR}=$ "concha rumo", $\mathrm{SG}=$ "señorita gigante", $\mathrm{AM}=$ "yuca amarilla", $\mathrm{CO}=$ "cogollo morado", $\mathrm{PL}=$ "paloma rumo", $\mathrm{TJ}=$ "tijerilla", $\mathrm{SA}=$ "sarayacu", $\mathrm{VI}=$ "vidrillo", $\mathrm{ZO}$ = "cola de zorro", PS = "pinsha rumo", TG = "tigre", $P B=$ "piririca del bajo", UN = "ungurahui", $P U=$ "pucallpina", $A R=$ "Arahuana rumo", $\mathrm{WA}=$ "wasahi rumo", $\mathrm{PN}=$ "palo negro", $\mathrm{BL}=$ "yuca blanca", $\mathrm{AP}=$ "arpón rumo", $\mathrm{BO}=$ "bolsa rumo", TR= "tres mesina", LC = "lacre rumo", TM= "tijerilla morada", PA = "piririca del alto". 


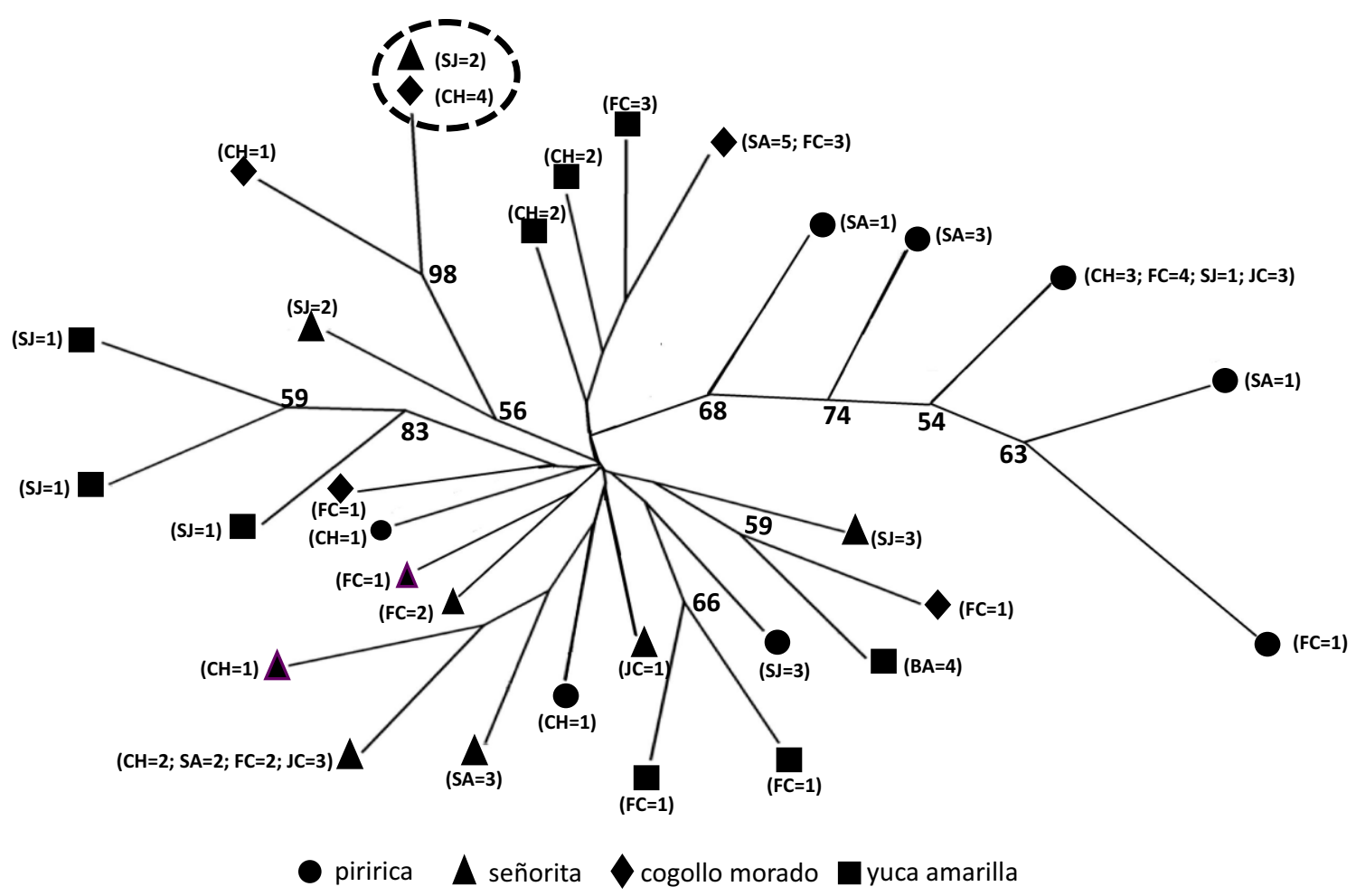

Figura 2. Dendrograma mostrando los resultados del análisis Neighbor Joining para las 4 etnovariedades comerciales de la baja del rio Ucayali. Abreviaciones: Localidades: $\mathrm{CH}=$ Chingana, $\mathrm{FC}=$ Flor de Castaña, $\mathrm{SA}=$ Sapuena, SJ= San José, BA= Bagazán. 


\section{BIBLIOGRAFÍA CITADA}

Acosta, R.A; Tamayo, A.C; Palacios, R. 2006. Caracterización morfológica y extracción de ADN de 11 clones de yuca (Manihot esculenta Crantz) en la universidad EARTH, Costa Rica. Tierra Tropical, 2 (1): 67-75.

Alzate, G.; Vallejo, C.; Ceballos, L.H.; Pérez, J.C.; Fregene, M. 2009. Variabilidad genética de la "yuca" cultivada por pequeños agricultores de la región Caribe de Colombia. Acta Agronómica, 59(4): 385-393.

Belkhir, K.; Borsa, P.; Chichi, I.; Raufast, N.; Bonhomme, F. 2004. GENETIX 4.05.2, logiciel sous windowns TM pour la genétique des populations. Laboratoire génome, populations, interactions, CNRS UMR 5000, Université de Montpellier II, Montpellier, France.

Ben-Har, A.; Charcosset, A.; Bourgoin, M.; Guiard, J. 1995. Relationships between genetic markers and morphological traits in a maize inbred lines collection. Euphytica, 84:145-154.

Boster, J.S. 1983. A comparison of the diversity of Jivaroan gardens with that of the tropical forest. Human Ecology. 11: 47-68.

Bravo, C. 2009. Caracterización morfológica y molecular de accesiones de maíz negro (Zea mays L.) mediante Análisis de Secuencias Simples Repetidas. Tesis de pre-grado. Universidad san francisco de Quito. 61pp.

Chavarriaga-Aguirre, P.; Maya, M.M.; Bonierbale, M.W.; Kresovich, S.; Fregene, M.A.; Tohme, J.; Kochert, G. 1998. Microsatellites in cassava (Manihot esculenta Crantz): Discovery, inheritance and variability. Theor Appl Genet., 97:493-501.

Crouch, H.; Crouch, J.; Madsen, S.; Vuylsteke, D.; Ortiz, R. 2000. Comparative analysis of phenotypic and genotypic diversity among plantain landraces (Musa spp., AAB group). Theor. Appl. Genet., 101: 1056-1065.

Demey, J.R; Zambrano, A.Y, Fuenmayor, F; Segovia V. 2003. Relación entre caracterizaciones molecular y morfológica en una colección de "yuca". Venezuela. INCI. 28(12): 684-689

Doyle, J.J.; Doyle, J.L. 1987. Isolation of plant DNA from fresh tissue. Focus, 12: 13-15.

Elias, M.; Mckey, D. 2000. The unmanaged reproductive ecology of domesticated plants in traditional agroecosystems: an example involving cassava and a call for data. Acta Oecol., 21:223-230.
Emperaire, L.; Pinton, F. 1998. Second, g. gestion dinamique de la diversité variétale du manioc en Amazonie du nord-ouest. Natures Sciences Sociétés, 6(2):27-42.

Felsenstein, J. 1993. Phylogeny Inference Package (PHYLIP). Version 3.5. University of Washington, Seattle. Washington. 132pp.

Fregene, M.; Roca, W.; Okogbenin, E.; Akano, A.; MBA, C.; Chavarriaga, A. 2001. Cassava's potencial in Asia in the 21st century present situation and future researsh and development needs. Centro Internacional de Agricultura Tropical (CIAT) and Institute of Agricultural Sciences of South Vietnam (IAS). Cali, Colombia. $150 \mathrm{pp}$.

Fukuda, W.M.G.; Guevara, C.L. 1998. Descritores morfológicos e Agronómicos para a caracterizacäo de mandioca (Manihot esculenta Crantz). Empresa Brasileira de Pesquisa Agropecuária (EMBRAPA), Bahia, BA. 38pp.

Hershey, C.H. 1994. Manihot genetic diversity In: Internacional network for cassava genetic resources. Internacional Crop Network Series (IPGRI), Rome, v.10. p. 111-134.

Iglesias, C.; Hernández, L.A.1994. Introducción de diversidad genética mejorada a nivel de campos de agricultores. En: Interfase entre los programas de mejoramiento, los campos de los agricultores y los mercados de la yuca en Latinoamérica. Centro Internacional de Agricultura Tropical CIAT, Cali, Colombia. 151-157pp.

Inga Sánchez H.; López Parodi J. 2001. Diversidad de yuca (Manihot esculenta Crantz) en Jenaro Herrera, Loreto - Perú. Instituto de Investigaciones de la Amazonía Peruana. Documento Técnico Nº 28.

Lobo, R. 2004. Caracterización de yuca (Manihot esculenta Crantz).En: Palma, R. Conservación in situ de cultivos nativos y parientes silvestres. Chosica, PE. Seminario taller. p. 136-169.

Mba, R.E.C.; Stephenson, P.; Edwards, K.; Melzer, S.; Nkumbira, J.; Gullberg, U.; et al. 2001. Simple sequence repeat (SSR) markers survey of the cassava (Manihot esculenta Crantz) genome: towards an SSR-based molecular genetic map of cassava. Theor. Appl. Genet., 102:21-31.

Mkumbira, J.; Chiwona-Karlturn, L.; Largercrantz, U.; Mahungu, N.; Saka, J.; Mhone, A.; Bokanga, M.; Brimer, L.; Gullberg, U.; Rosling, H. 2003. Classification of cassava into 'bitter' and 'cool' in Malawi: from farmers' perception to characterisation by molecular markers. Euphytica, 132:7-22. 
Mühlen, G.S.; Martins, P.S.; Ando, A. 2000. Variabilidade genética de etnovariedades de mandioca, avaliada por marcadores de DNA. Sci Agric., 57:319-328.

Nei M. 1972. Genetic Distance between Populations. American Naturalist., 106: 283292.

Nye, M.M. 1991. Mis-measure of manioc (Manihot esculenta, Euphorbiaceae). Economic Botany, 45: 47-57.

Ortiz, R. 1997. Morphological variation in Musa germplasm. Genet. Res. Crop. Evol., 44: 393404

Peakall, R.; Smouse, P.E.; 2006. GENALEX 6: Genetic analysis in excel. Population genetic software for teaching and research. Molecular ecology Notes, 6:288-295.

Peroni, N.; Martins, P.S.; Ando, A. 1999. Diversidade inter e intra-específica e uso de análise multivariada para morfologia da mandioca (Manihot esculeta Crantz): um estudo de caso. Scientia Agricola, 56(3): 587-595.

Peroni, N.; Martins, P.S. 2000. Influência da dinâmica agrícola itinerante na geração de diversidade de etnovariedades cultivadas vegetativamente. Interciencia, 25(1):22-29.
Pujol B.; Gigot, G.; Laurent, G.; Pinheiro-Kluppel, M.; Elias, M.; Mckey, H.M.; Mckey, D. 2005. Germination ecology of cassava (Manihot esculenta Crantz, Euphorbiaceae) in traditional agroecosystems: Seed and seedling biology of a vegetatively propagated domesticated plant. Econ. Bot., 56: 366-379.

Sardos, J.; Mackey, E.; Duval, M.F.; Malapa, R.; Noyer, J.L.; Lebot, V. 2008. Evolution of cassava (Manihot esculenta Crantz) after recent introduction into a South Pacific Island system: the contribution of sex to the diversification of a clonally propagated crop. Genome, 51:912-921.

Valadez-Moctezuma, E.; Kahl, G.; Ramser, J.; Hüttel, B.; Rublúo-Islas, A. 2001. Técnicas moleculares para la caracterización de genomas vegetales (Garbanzo) y algunas aplicaciones potenciales. Fitotec. Mex., 24(1): 103-120.

Vaz, M.; Satovic, Z.; Pego, S.; Fevereiro, P. 2004. Assessing the genetic diversity of Portuguese maize germplasm using microsatellite markers. Euphytica, 137: 63-72.

Recibido: 05 de marzo del 2015

Aceptado para publicación: 23 mayo del 2015 